\title{
Miniatur-Stehende-Welle-Interferometer auf Basis schneller, transparenter Photodioden
}

\author{
Ingo Ortlepp ${ }^{1}$, Hans-Joachim Büchner ${ }^{1}$, Tzvetan Ivanov ${ }^{2}$, Manuel Hofer ${ }^{2}$, Jens-Peter Zöllner ${ }^{2}$, \\ Ivo Rangelow ${ }^{2}$, Eberhard Manske ${ }^{1}$ \\ ${ }^{1}$ Institut für Prozessmess- und Sensortechnik, Technische Universität IImenau, D-98693 IImenau, \\ info.pms@tu-ilmenau.de, Tel.: +49 $367769-2822$ \\ ${ }^{2}$ Fachgebiet Mikro- und nanoelektronische Systeme, Technische Universität IImenau, D-98693 IImenau, \\ jens-peter.zoellner@tu-ilmenau.de,Tel.: +49 3677 69-3717
}

\section{Zusammenfassung}

Um den Anforderungen an die Miniaturisierung von Messsystemen Rechnung zu tragen, wurde an der TU IImenau ein neuartiges Interferometer auf Grundlage von optischen stehenden Wellen entwickelt. Das Interferometerprinzip erlaubt einen einfachen, linearen und kompakten Interferometeraufbau. Die für das zu Grunde liegende Prinzip erforderlichen dünnen, transparenten Photodioden können durch den Einsatz von Standard-Halbleitertechnologien unkompliziert in großen Stückzahlen hergestellt werden. Im Folgenden werden das Funktionsprinzip des Interferometers, die Herstellung der Photodioden sowie die durchgeführten messtechnischen Untersuchungen dargelegt.

Keywords: Interferometer, optische stehende Welle, transparent, Photodiode

\section{Einleitung}

Für Aufgaben der Längenmesstechnik entwickelte Laserinterferometer werden mit hoher Leistungsfähigkeit in der Industrie, in der Halbleitertechnologie und der Präzisionsmesstechnik eingesetzt [1, 2]. Der Aufbau der kommerziellen Laserinterferometer ist sehr komplex, erfordert eine manuelle Montage, umfangreiche Justage und ist dadurch sehr kostenintensiv. Weiterhin sind, auf Grund des Aufbaus mit gekreuztem Mess- und Referenzstrahl beim üblicherweise eingesetzten Michelson-Interferometer (Abb. 1), der Miniaturisierung enge Grenzen gesetzt.

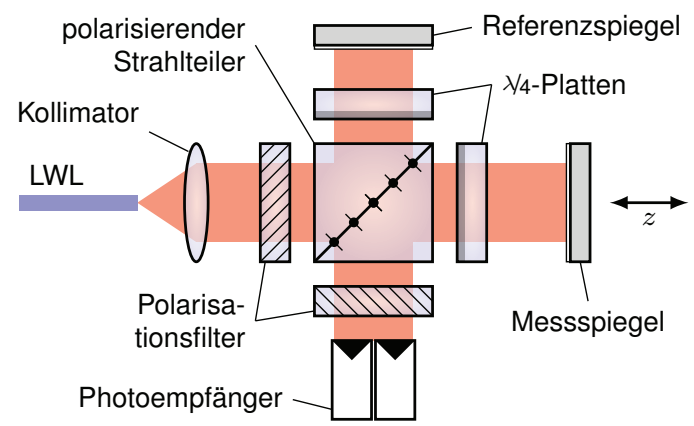

Abb. 1: Michelson-Interferometer in industrieller Ausführung

Das Stehende-Wellen-Interferometer stellt ein völlig neues lineares Interferometerkonzept dar, das auf der Interferenz von Strahlen entgegengesetzter Ausbreitungsrichtung (optische stehende Welle) beruht. Mit diesem Ansatz ist es möglich, Interferometer mit einer einfachen, linearen und kompakten Struktur aufzubauen, welche lediglich aus der Laserquelle, einem ultradünnen, transparenten Photosensor und dem Messspiegel bestehen. Daraus resultiert ein enormes Potenzial für Kosteneinsparungen und zur extremen Miniaturisierung laserinterferometrischer Messanordnungen.

\section{Grundlagen}

\subsection{Die stehende Welle}

Bei der Reflexion einer optischen Welle an einem Spiegel interferieren einfallender und reflektierter Strahl in entgegengesetzter Richtung $[3,4]$. Für die elektrische Feldstärke der einfallenden Welle $E_{\text {in }}$ gilt

$$
E_{\text {in }}=A_{\text {in }} e^{j \frac{2 \pi}{\lambda}(z-c t)}
$$

mit der Amplitude $A_{\text {in }}$, der komplexen Einheit $j$, der Wellenlänge $\lambda$, der Lichtgeschwindigkeit $c$, der Zeit $t$ und der Ausbreitungsrichtung $z$. Die reflektierte Welle erfährt bei der Reflexion einen Phasensprung von $\pi$ gegenüber der einfallenden Welle, daher gilt für die Feldstärke der reflektierten Welle $E_{\mathrm{re}}$

$$
E_{\mathrm{re}}=A_{\mathrm{re}} e^{j \frac{2 \pi}{\lambda}\left(-z-c t+\frac{\lambda}{2}\right)}
$$

Die Feldstärke der stehenden Welle resultiert aus der Superposition der Teilfeldstärken. Für einen idealen Spiegel gilt für die Intensität $I_{\mathrm{sw}}$ der stehenden Welle

$$
I_{\mathrm{sw}}=2 I_{0}\left(1-\cos \left(\frac{4 \pi}{\lambda} z\right)\right)
$$

mit der Intensität $I_{0}$ des einfallenden Strahls. Die Intensität $I_{\mathrm{sw}}$ variiert zwischen 0 (destruktive 
Interferenz) und $4 I_{0}$ (konstruktive Interferenz). Dabei sind die Intensitätsminima und -maxima auf Grund des konstanten Phasensprungs von $\pi$ mit der Spiegeloberfläche fest verkoppelt. Das Intensitätsprofil der stehenden Welle kann nun von einem Photosensor abgetastet werden, welcher zu diesem Zweck transparent und deutlich dünner als die optische Wellenlänge sein muss [5]. Werden der Messspiegel und der Sensor in $z$-Richtung relativ zueinander bewegt, verschiebt sich das den Sensor durchsetzende Intensitätsprofil, wodurch ein sinusförmiges Sensorsignal entsteht. Zur Richtungserkennung ist ein zweiter Sensor im Abstand von $(2 k-1) \frac{\lambda}{8}, k \in \mathbb{Z}$ erforderlich. Mit dieser Anordnung werden zwei Sensorsignale mit einer Phasenverschiebung von $90^{\circ}$ erzeugt, wodurch eine Vor-RückwärtsZählung ermöglicht wird (Abb. 2).

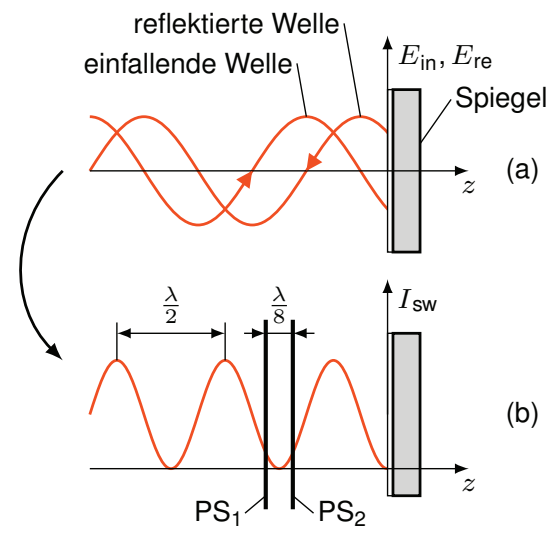

Abb. 2: (a) Feldstärken $E_{i n}, E_{r e}$ der einfallenden und reflektierten Welle.

(b) Intensitätsprofil $I_{s w}$ der stehenden Welle. Abtastung mit Photosensoren PS1 und PS2.

Ein auf dem beschriebenen Prinzip basierendes Interferometer besitzt ein beachtliches Miniaturisierungspotenzial. Durch den einfachen linearen Aufbau und die geringe Anzahl notwendiger Bauelemente ist der erforderliche Bauraum nur geringfügig größer als der Durchmesser des Messstrahls (Abb. 3).

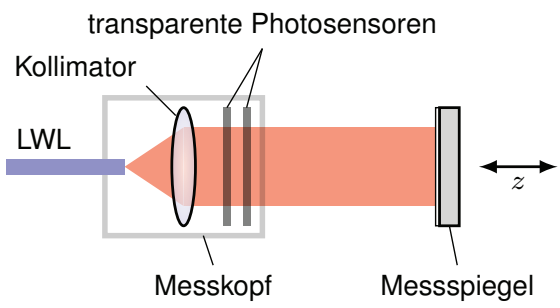

Abb. 3: Prinzipieller Aufbau eines StehendeWelle-Interferometers. Die Komplexität ist deutlich geringer als die eines MichelsonInterferometers (vgl. Abb. 1).

\subsection{Sensoreigenschaften}

Da sich der Photosensor zur Erfassung der Intensität in der stehenden Welle befindet, müssen für eine adäquate Funktion des Interferometers die im Folgenden beschriebenen Anforderungen erfüllt sein.

Zunächst muss der Photosensor über eine entsprechende Transparenz verfügen, damit die Feldstärken $E_{\text {in }}$ der einfallenden und $E_{\mathrm{re}}$ der reflektierten Welle annähernd die gleiche Amplitude aufweisen. Andernfalls entsteht mit sinkender Transparenz ein wachsender, unerwünschter Offset $s_{-}$des Sensorsignals, während der erwünschte Wechselanteil $s_{\sim}$ abnimmt.

Die zweite Eigenschaft des Photosensors ist die Dicke $d$ der aktiven, photoempfindlichen Schicht. Da die stehende Welle die gesamte aktive Schicht durchdringt, erfolgt eine Integration des Intensitätsprofils innerhalb der Schichtgrenzen. Für die Dicke der aktiven Schicht existieren zwei Extremfälle. Zum einen eine Dicke $d=0$ bei der kein Sensorsignal erzeugt wird und zum anderen eine Dicke $d=k \frac{\lambda}{2}$, bei der über eine bzw. mehrere Perioden des Intensitätsprofils integriert wird, wodurch das Sensorsignal allein aus einem Gleichanteil $s_{-}$ohne nutzbaren Wechselanteil $s_{\sim}$ besteht. Die Wahl der Schichtdicke ist daher ein Kompromiss zwischen Signalkontrast (Verhältnis $s_{\sim}$ zu $s_{-}$) und Amplitude des Wechselsignals $s_{\sim}$. Unter Berücksichtigung der Absorption ist die optimale Dicke der aktiven Schicht $d=\frac{\lambda}{4}$, was die besondere Herausforderung bei diesem Interferometerprinzip darstellt.

Die dritte Eigenschaft ist der Reflexionsgrad $R$ des Sensors. Prinzipbedingt muss der Sensor senkrecht zur $z$-Achse und damit parallel zur Messspiegeloberfläche ausgerichtet werden. Dadurch entsteht ein optischer Resonator (FabryPérot-Aufbau) zwischen Sensor und Messspiegel, welcher die ursprüngliche stehende Welle durch Mehrfachreflexionen beeinflusst. Dies hat zur Folge, dass der Signalkontrast abnimmt und das Sensorsignal durch Harmonische verzerrt wird. Um diese negativen Einflüsse zu minimieren, ist eine hinreichende Entspiegelung des Photosensors notwendig.

\subsection{Stand der Technik}

Stehende Wellen und der Phasensprung an reflektierenden Oberflächen wurden erstmals von WIENER [6] untersucht. Die Anwendung von stehenden Wellen zur Längenmessung wird erstmals von BÜCHNER [7] beschrieben. In diesem Patent wird der grundlegende Aufbau eines Stehende-Welle-Interferometers mit Laserquelle, Messspiegel und zwei hintereinander angeordneten, $90^{\circ}$-phasenverschobenen Sensoren erläutert. 
Für die Form und Funktionsweise des Photosensors existieren verschiedene Ansätze. Einen Sensor auf Basis eines Photoelektronenvervielfachers stellen SILVERTOOTH und JACOBS in [8] vor. Der Sensor enthält als photoaktive Schicht eine Photokathode mit einer Dicke von $50 \mathrm{~nm}$.

Die Anwendung eines Stehende-WelleSensors in einem Fourier-Spektrometer wird von KUNG u. a. in [9] beschrieben. Der eingesetzte Sensor ist ein Photowiderstand, welcher durch Abscheiden von amorphem Silizium auf einen Quarzträger hergestellt wird.

In [10] und [11] beschreiben SASAKI u.a. einen transparenten Silizium-Photosensor auf einem Quarzträger. Bei diesem Photosensor sind zwei Photodioden nebeneinander angeordnet. Um die notwendige Phasenverschiebung von $90^{\circ}$ zu erreichen, wird der Träger im Bereich einer der Photodioden lokal abgedünnt, was eine Änderung der optischen Weglänge von $\frac{\pi}{2}$ zur Folge hat.

In einer gemeinsamen Forschungsarbeit des Forschungszentrums Jülich und der TU IImenau wurde von BUNTE u.a. [12], [13] und MANDRYKA u. a. [14], [15] ein Photosensor entwickelt, welcher durch Gasphasenabscheidung von Silizium auf einen Glasträger hergestellt wird. Der Abscheideprozess wird so ausgeführt, dass durch sukzessives Aufbringen der entsprechenden Lagen zwei übereinandergeschichtete p-i-n-(positivintrinsisch-negativ)-Photodioden entstehen. Die Phasenverschiebung zwischen beiden Photodioden und die Entspiegelung des Gesamtsensors wird dabei durch eine entsprechende Wahl der einzelnen Schichtdicken erreicht.

LAZAR u.a. [16], [17] beschreiben einen Sensor, der aus einem Quarzglassubstrat, einer aktiven Schicht aus polykristallinem Silizium und mehreren Antireflexionsschichten besteht. Der Sensor wird zur gleichzeitigen Positionsmessung in einem optischen Resonator und der Laserwellenlängenstabilisierung auf diesen Resonator verwendet.

\section{Funktionsprinzip}

Zielsetzung bei Entwicklung der transparenten Photosensoren war neben dem Einsatz von Standard-Halbleitertechnologien eine Grenzfrequenz größer als $4 \mathrm{MHz}$, um die Sensoren auch für Messgeschwindigkeiten über $1 \mathrm{~m} \mathrm{~s}^{-1}$ einsetzen zu können. Der Photosensor ist daher als laterale $p$-i-n-Photodiode mit einem streifenförmigen Dotierungsprofil ausgeführt (Abb. 4 und 5). Als Substrat dienen handelsübliche Silicon-On-Insulator-(SOI)-Wafer, in deren obere Siliziumschicht das $\mathrm{p}$-i-n-Profil dotiert wird. Um eine hinreichende Transparenz zu erreichen, wird das Trägermaterial des Wafers von der Rückseite her lokal abgeätzt. Auf diese Weise lässt sich eine ultradünne, transparente, photoelektrisch aktive Membran herstellen. Die Fläche des transparenten, aktiven Bereichs folgt aus dem Durchmesser des Laserstrahls $(l=1 \mathrm{~mm})$, die Dicke der photoaktiven Schicht ist für Silizium mit einer Brechzahl $n=3,8$ und einer Laserwellenlänge $\lambda_{0}=633 \mathrm{~nm}$

$$
d=\frac{\lambda_{0}}{4 n}=42 \mathrm{~nm} .
$$

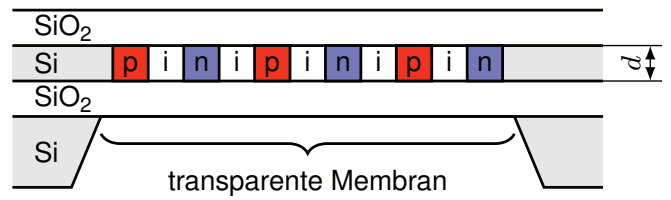

\section{Abb. 4: Schichtaufbau des Grundsensors (Schnittansicht). Kontaktierung nicht dargestellt.}

Die Breite $w$ der dotierten Bereiche beeinflusst einerseits die Ladungsträgerbeweglichkeit und damit die Photoempfindlichkeit sowie andererseits die Sperrschichtkapazität und damit die Grenzfrequenz des Sensors. Für die verwendete Technologie ist die optimale Breite $w=10 \mu \mathrm{m}$.

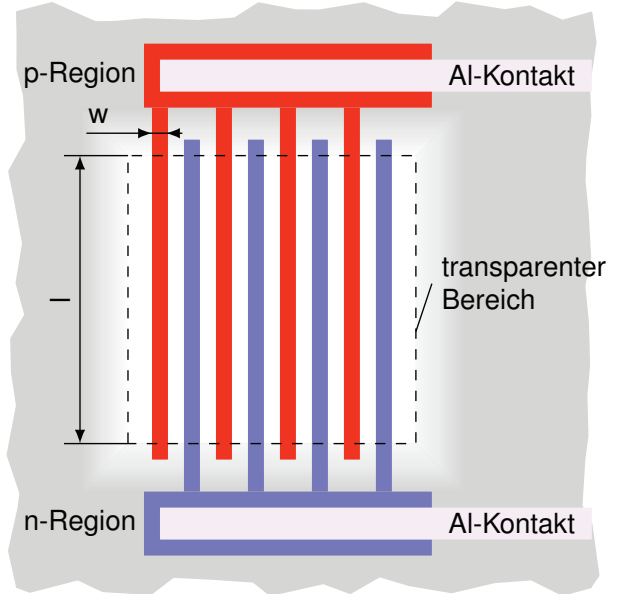

Abb. 5: Ansicht des Sensors in Strahlrichtung.

Der Sensor wird als Photodiode mit negativer Vorspannung betrieben. Bei Lichteinfall werden in den i-Gebieten Ladungsträger generiert, welche über die $\mathrm{p}$ - bzw. n-Gebiete abfließen und einen Photostrom erzeugen. Bei Betrieb in der stehenden Welle entsteht bei einer Relativbewegung zwischen Sensor und Messspiegel somit ein sinusförmiger Photostrom $i$, bestehend aus einem konstanten Offset $i_{-}$und einem Wechselsignal $i_{\sim}$.

\section{Sensorherstellung}

Für die Herstellung der Sensoren werden SOI-Wafer mit einer Si-Schichtdicke von $100 \mathrm{~nm}$ - 
$200 \mathrm{~nm}$ verwendet. Zunächst erfolgt eine vollflächige Oxidation der oberen Si-Schicht des Wafers. Auf diese Weise wird die Dicke der photoaktiven Schicht auf das erforderliche Maß eingestellt. Weiterhin besitzt die $\mathrm{SiO}_{2}$-Schicht die Funktion eines Streuoxids für die nachfolgende Dotierung der $\mathrm{p}$ - und $\mathrm{n}$-Gebiete. Dabei werden die Dotierbereiche mittels Photolithographie definiert und Phosphor bzw. Bor mit Hilfe einer lonenimplantation eingebracht. Abschließend werden die dotierten Bereiche mit einer Aluminiumschicht kontaktiert. Nach diesem Prozessschritt ist der Sensor photoelektrische funktionsfähig.

Um Vielstrahlinterferenzen zwischen der Sensorfläche und dem Messspiegel zu vermeiden, muss der Reflexionsgrad des Sensors entsprechend gering sein. Der favorisierte Ansatz, durch eine geeignete Wahl der einzelnen Schichtdicken des Grundsensors eine immanente Entspiegelung zu erreichen, ist mit der verwendeten Technologie nicht möglich. Daher ist der Auftrag zusätzlicher Antireflexionsschichten notwendig. Diese wurden mittels physikalischer Gasphasenabscheidung (PVD) auf beiden Seiten der Sensormembran aufgebracht. Auf diese Weise konnte der rechnerische Reflexionsgrad von $R=49,8 \%$ (unbeschichteter Sensor) auf $R=0,06 \%$ (beschichteter Sensor mit angepassten Grundschichten) reduziert werden. Abbildung 6 zeigt den Schichtaufbau des fertigen Sensors.

$\left.\begin{array}{|ll|}\hline \text { Glas + Kleber } & n=1.51 \\ \hline \text { AR-Schicht 1 } & n=3.94 \\ \hline \mathrm{SiO}_{2} & n=1.46 \\ \hline \mathrm{Si} \text { (aktive Schicht) } & n=3.87 \\ \hline \mathrm{SiO}_{2} & n=1.46 \\ \hline \text { AR-Schicht 2 } & n=2.11 \\ \hline \text { Luft } & n=1\end{array}\right\} \begin{aligned} & \text { Grund- } \\ & \text { sensor }\end{aligned}$

Abb. 6: Sensorkonfiguration mit zwei zusätzlichen AR-Schichten und angepasstem Grundsensor. Die Brechzahlen der AR-Schichten sind theoretische Werte, der berechnete Reflexionsgrad beträgt $R=0,06 \%$.

Um eine hinreichende Transparenz zu erreichen, muss das rückseitige Substratmaterial im Bereich der aktiven Fläche vollständig entfernt werden, wodurch eine dünne, transparente Membran entsteht. Die Herstellung dieser Membran stellt eine besondere Herausforderung zur Umsetzung dieses Interferometerprinzips dar. Da die Intensität $I_{\mathrm{sw}}$ der stehenden Welle über das gesamte Volumen der aktiven Schicht integriert wird, muss die Sensormembran eine entsprechende Ebenheit aufweisen. Andernfalls umfasst der generierte Photostrom verschiedene Phasenwinkel der stehenden Welle, wodurch der erwünschte Wechselanteil $i_{\sim}$ sinkt. Abhängig von der Gestalt der Verformung entfällt der Wechselanteil bei einer Ebenheitsabweichung von ca. $\lambda / 2$ vollständig, da der Photostrom in diesem Fall eine Integration über eine volle $\mathrm{Pe}$ riode von $I_{\mathrm{sw}}$ darstellt. Für einen ausreichenden Signalkontrast beträgt die zulässige Ebenheitsabweichung daher $\lambda / 4$. Unter Berücksichtigung der Membrangesamtdicke von lediglich $600 \mathrm{~nm}$ (aktive Schicht und passive $\mathrm{SiO}_{2}$-Schichten) und der Membranfläche von $1 \mathrm{~mm} \times 1 \mathrm{~mm}$ ist daher eine zusätzliche mechanische Stabilisierung der Membran erforderlich. Diese wird durch eine Glasplatte erreicht, welche vor dem Entfernen des rückseitigen Substratmaterials auf die Vorderseite des Sensors aufgeklebt wird, um die Membran in ihrem ebenen Ausgangszustand zu fixieren. Anschließend wird das Substratmaterial im Bereich der aktiven Fläche mittel reaktivem lonenätzen vollständig entfernt. Die Vorder- und Rückseite des Sensors zeigt Abb. 7. Die Abmessungen des Photosensors sind ca. $5 \mathrm{~mm} \times 7 \mathrm{~mm} \times 0.5 \mathrm{~mm}$, der aktive Bereich und die lokale Abdünnung auf der Rückseite haben eine Fläche von jeweils ca. $1 \mathrm{~mm} \times 1 \mathrm{~mm}$.

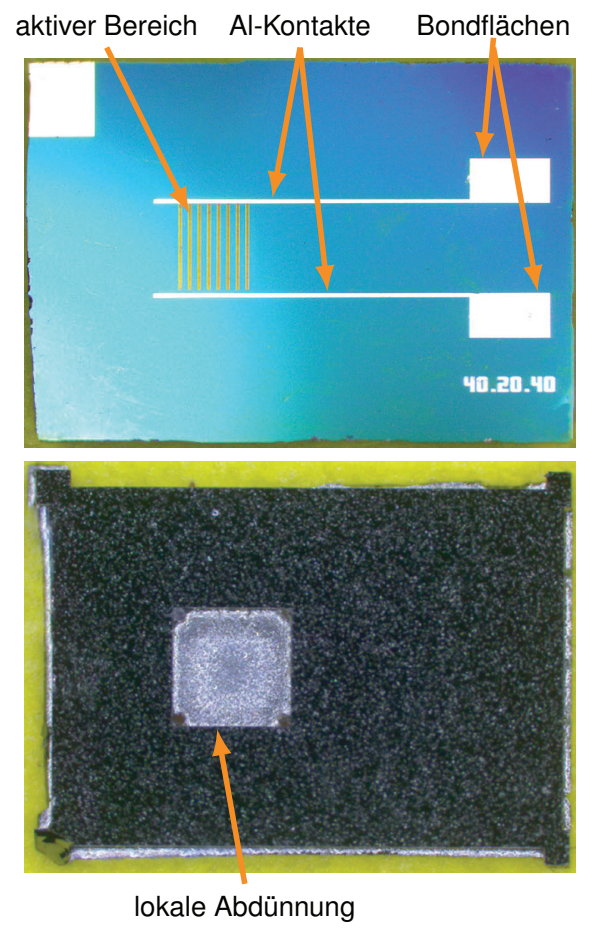

Abb. 7: Bilder des Sensors vor dem Aufkleben der Glasplatte zur Stabilisierung der Membran. Auf der Vorderseite (oben) sind die streifenförmigen Dotiergebiete auf der noch nicht vollständig abgedünnten Membran erkennbar. Die Rückseite (unten) ist lokal bis auf ca. $20 \mu \mathrm{m}$ vorabgedünnt.

Schwerpunkt bei der Gestaltung des Herstellungsprozesses war, überwiegend Standard- 
Halbleiterprozesse einzusetzen, die jeweils auf einen gesamten Wafer angewendet werden können. Auf diese Weise lässt sich eine große Anzahl Sensoren kostengünstig herstellen, was den möglichen Einsatzbereich von interferometrischen Messverfahren auf kostensensitive Messaufgaben erweitert.

\section{Experimenteller Nachweis}

\subsection{Einzelsensor}

Eine grundlegende Eigenschaft der Sensoren ist deren Diodenverhalten. Dieses wurde nach jedem Prozessschritt ab dem Aufbringen der Aluminiumkontaktierung überprüft. Ziel war es, anhand der Diodenkennlinie eventuelle negative Prozesseinflüsse zu identifizieren und ggf. Prozesse entsprechend anzupassen. Die fertiggestellten Sensoren zeigen ein gutes Diodenverhalten mit einem geringen Sperrstrom von ca. $1 \mathrm{nA}$.

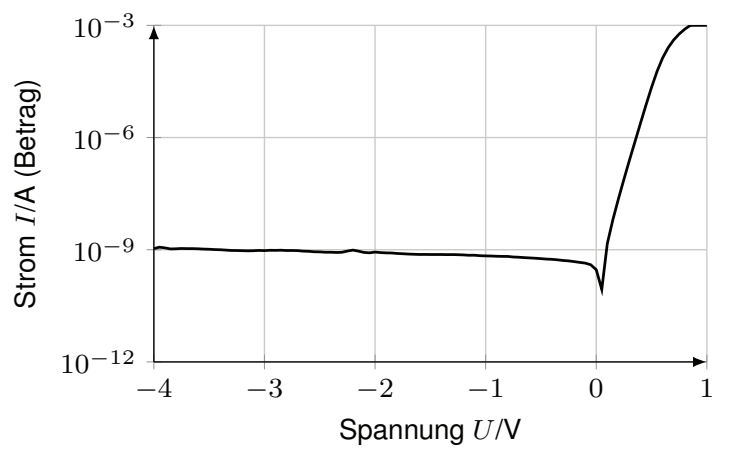

Abb. 8: Diodenkennlinie des unbeleuchteten Sensors. Der Sperrstrom beträgt ca. $1 \mathrm{nA}$.

Der Einsatz der Sensoren in einem Stehende-Welle-Interferometer stellt ferner Anforderungen hinsichtlich der erreichbaren Grenzfrequenz. Bei einer Relativbewegung zwischen Sensor und Messspiegel entlang der optischen Achse entsteht ein geschwindigkeitsabhängiges Wechselsignal $i_{\sim}$. Dieses Signal muss bei allen auftretenden Geschwindigkeiten mit der entsprechenden Amplitude vorliegen. Für den Großteil der Messaufgaben ist dabei eine Geschwindigkeit von $1 \mathrm{~m} \mathrm{~s}^{-1}$ ausreichend. Für eine Laserwellenlänge von $633 \mathrm{~nm}$ resultiert daraus eine Messfrequenz $f=3 \mathrm{MHz}$. Die Grenzfrequenz der Sensoren wurde jedoch auf Grund der aufwändigen Realisierung hoher Spiegelgeschwindigkeiten nicht in einem Stehende-WelleAufbau, sondern mittels einer modulierbaren Laserdiode ermittelt (siehe Abb. 9). Um den Frequenzgang der Laserdiode zu korrigieren, enthält der Versuchsaufbau einen Referenzempfänger mit $200 \mathrm{MHz}$ Bandbreite. Zur Auswertung wurde ein linearer Frequenzgang des Referenzempfängers angenommen und das Verhältnis der Signalamplitude des untersuchten Sensors zur Signal- amplitude der Referenz ermittelt. Die Sensoren

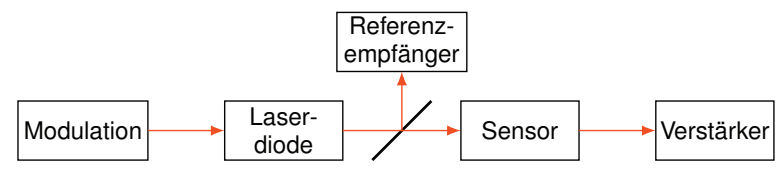

Abb. 9: Aufbau zur Messung des Frequenzgangs. Der Referenzempfänger dient der Korrektur des Frequenzgangs der Laserdiode.

weisen Grenzfrequenzen bis $70 \mathrm{MHz}$ auf, was den Einsatzbereich auf Messgeschwindigkeiten über $20 \mathrm{~m} \mathrm{~s}^{-1}$ erweitert. Abbildung 10 zeigt beispielhaft den Frequenzgang eines Sensors.

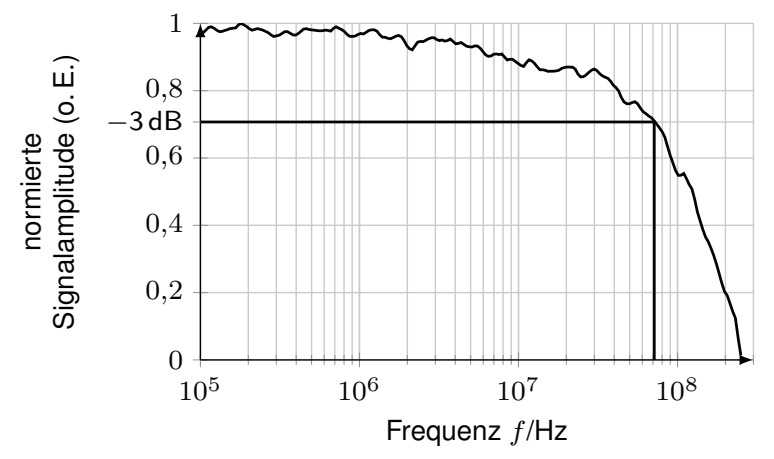

Abb. 10: Frequenzgang eines Sensors, gemessen mittels modulierbarer Laserdiode. Die $-3 \mathrm{~dB}$-Frequenz beträgt $71 \mathrm{MHz}$.

\subsection{Doppelsensor}

Nachdem die grundlegende Funktionsfähigkeit der Sensoren nachgewiesen werden konnte, wurde ein Doppelsensor in Hybridbauweise aufgebaut. Dieser besteht aus zwei einzelnen justierbaren Sensoren, deren longitudinaler Abstand mit Hilfe eines Piezoaktors eingestellt wird. Auf diese Weise kann der Phasenwinkel zwischen beiden Sensorsignalen auf den erforderlichen Wert von $90^{\circ}$ eingestellt und ggf. nachjustiert werden. Der Doppelsensor wurde anschließend in einen Stehende-Welle-Aufbau integriert und untersucht. Abbildung 11 zeigt schematisch den verwendeten Aufbau.

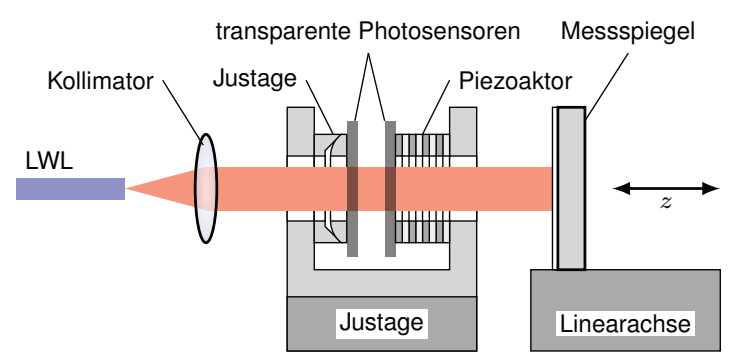

Abb. 11: Stehende-Welle-Doppelsensor in $\mathrm{Hy}$ bridbauweise mit Justage zur senkrechten Ausrichtung der Sensoren zum Laserstrahl und Piezoaktor zur Einstellung des Abstandes auf $(2 k-1) \frac{\lambda}{8}$. 
Als Laserquelle wird ein fasergekoppelter $\mathrm{He}-\mathrm{Ne}$-Laser verwendet. Prinzipbedingt muss der Messspiegel senkrecht zu dem Laserstrahl ausgerichtet werden, wodurch das vom Spiegel reflektierte Licht nach Durchgang durch die beiden Sensoren in die Laserquelle zurückgekoppelt wird. Um diese Rückreflexionen und damit eine Störung der Laserquelle zu unterdrücken, wurde ein Faraday-Isolator zwischen $\mathrm{He}-\mathrm{Ne}$-Laser und Fasereinkopplung eingesetzt (siehe Abb. 12).

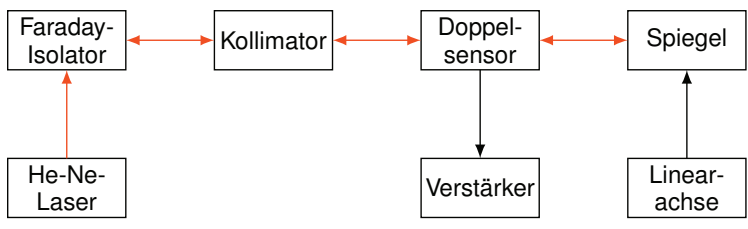

Abb.12: Aufbau eines Stehende-WelleInterferometers mit einem Doppelsensor. Die Laserquelle ist ein fasergekoppelter $\mathrm{He}-\mathrm{Ne}$-Laser. Die Linearachse bewegt den Messspiegel entlang $z$.

Nach dem Platzieren aller Bauteile erfolgte die Justage der Sensoren und des Messspiegels auf ein maximales Messsignal $i_{\sim}$. Anschließend wurde die Linearachse mit einer Geschwindigkeit $v=0,5 \mathrm{~mm} \mathrm{~s}^{-1}$ verfahren und das Signal des Sensors erfasst.

Um die Signalqualität zu beurteilen und die vorhandenen Harmonischen zu bewerten, wurde eine Fouriertransformation (FFT) des Senorsignals durchgeführt (siehe Abb. 13). Dazu wurde der Teil des Signals zwischen den Beschleunigungs- und Bremsphasen des Messspiegels verwendet.

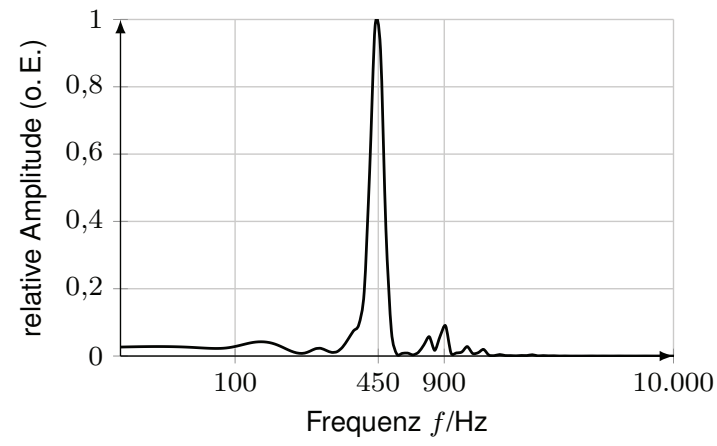

Abb. 13: Frequenzspektrum des Sensorsignals bei bewegtem Messspiegel in einem Stehende-Welle-Aufbau mit einer Grundfrequenz von $450 \mathrm{~Hz}$. Die Harmonische bei $900 \mathrm{~Hz}$ deutet auf Vielstrahlinterferenzen hin.

Die FFT zeigt erwartungsgemäß eine Grundschwingung bei $450 \mathrm{~Hz}$. Weiterhin ist eine Harmonische bei $900 \mathrm{~Hz}$ vorhanden, welche auf Vielstrahlinterferenzen hindeutet. Ursache dafür sind Reflexionen an der Sensoroberfläche, wel- che auf Grund der Fertigungstoleranzen bei der AR-Beschichtung nicht vollständig unterdrückt werden konnten.

Die entwickelten Sensoren sollen für Messgeschwindigkeiten bis $1 \mathrm{~m} \mathrm{~s}^{-1}$ eingesetzt werden. Für die Einzelsensoren erfolgte die Untersuchung der Grenzfrequenz wie beschrieben mittels modulierbarer Laserdiode. Für den StehendeWelle-Doppelsensor wurde hingegen der in Abb. 12 und 14 dargestellte Aufbau bestehend aus einer Laserquelle, dem Doppelsensor und einer den Messspiegel bewegenden Linearachse verwendet.

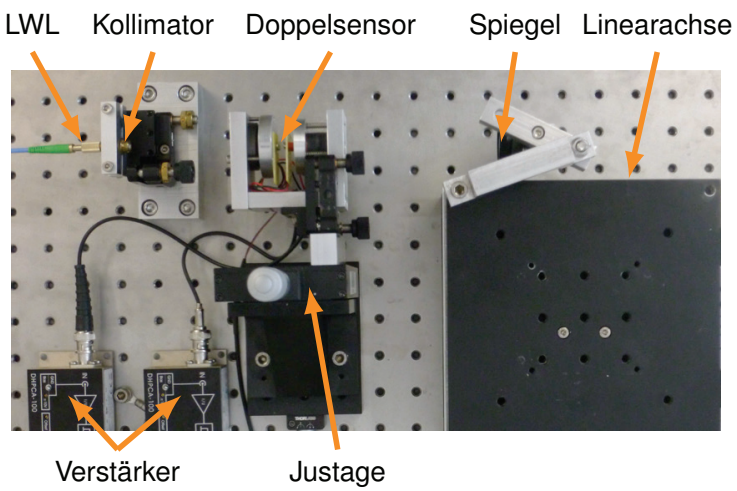

Abb. 14: Stehende-Welle-Aufbau mit Doppelsensor in Hybridbauweise. Die Justage dient der senkrechten Ausrichtung des Doppelsensors zum Laserstrahl.

Während der Messung wurde die Linearachse mit der maximalen Geschwindigkeit von $v=100 \mathrm{~mm} \mathrm{~s}^{-1}$ alternierend über den vollen Bewegungsbereich von $100 \mathrm{~mm}$ verfahren und die Sensorsignale aufgezeichnet. Abbildung 15 zeigt den aus den Signalen des Doppelsensors ermittelten Weg- und Geschwindigkeitsverlauf des Messspiegels. Mit der durchgeführten Messung konnte die Eignung des Doppelsensors für Messgeschwindigkeiten bis $100 \mathrm{~mm} \mathrm{~s}^{-1}$ nachgewiesen werden.

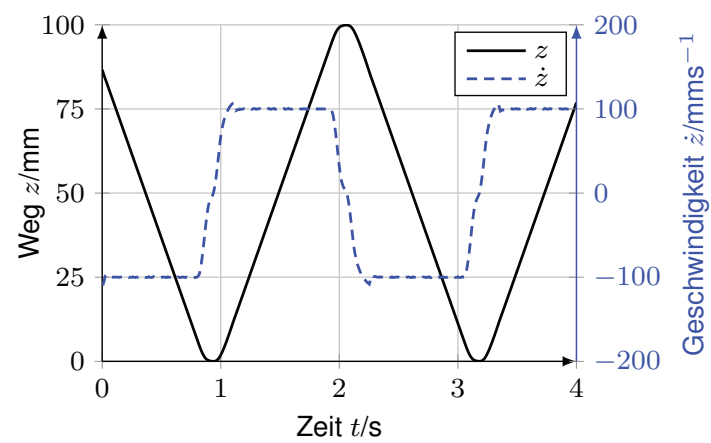

Abb. 15: Aus den Signalen des Doppelsensors ermittelter Weg- und Geschwindigkeitsverlauf beim Verfahren der Linearachse in einer alternierenden Bewegung. 


\section{Zusammenfassung und Ausblick}

Ein neuartiges Interferometerkonzept auf Basis einer optischen stehenden Welle und schnellen, transparenten Photosensoren wurde vorgestellt. Die hergestellten Sensoren sind als $\mathrm{p}$-i-n-Photodiode ausgebildet und können überwiegend mit Standard-Halbleiterprozessen hergestellt werden. Sie zeigen ein gutes photoelektrisches Verhalten (Diodencharakteristik, Photoempfindlichkeit, Linearität) und haben eine ausgezeichnete Dynamik, wodurch sie sich auch für Messgeschwindigkeiten $>1 \mathrm{~m} \mathrm{~s}^{-1}$ eignen. Die Eignung der Sensoren für den Einsatz in einem Stehende-Welle-Interferometer konnte in verschiedenen Messungen gezeigt werden Nachgewiesen wurden sowohl die grundlegende Funktion der Längenmessung, die Qualität der Sensorsignale sowie die Auswertung der Längenbzw. Positionssignale. Weiterhin konnte der Einsatz der Sensoren für Messungen über größere Messbereiche (>100 mm) und bei hohen Verfahrgeschwindigkeiten $\left(>100 \mathrm{~mm} \mathrm{~s}^{-1}\right)$ demonstriert werden.

Zukünftige Arbeiten werden als ersten Schritt die Miniaturisierung des Hybridsensors sowie im weiteren Verlauf die Konzeption und Herstellung eines integrierten Doppelsensors sowie eine verbesserte AR-Beschichtung zur Erhöhung der Signalqualität umfassen.

\section{Förderung}

Dieses Projekt wurde vom Bundesministerium für Bildung und Forschung unter dem Förderkennzeichen 03V0235 gefördert.

\section{Literatur}

[1] Theo AM Ruijl, und Jan van Eijk: „A Novel Ultra Precision CMM based on Fundamental Design Principles." in Proceedings of the ASPE summer conference on coordinate measuring machines, Charlotte, North Carolina, USA. 2003.

[2] G. Jäger, E. Manske, T. Hausotte und H.J. Büchner: „Nanomeasuring and nanopostioning Engineering" in Fringe - The 6th International Workshop on Advanced Optical Metrology, S. 390-397, Invited paper, 2009. doi:10.1016/j.measurement.2010.04.008

[3] E. Hecht: „Optik“ 5. Aufl., Oldenbourg Wissenschaftsverlag $\mathrm{GmbH}, 2009$.

[4] F. Graham Smith und Terry A. King: „Optics and photonics: an introduction" 2. Aufl., John Wiley \& Sons Ltd., 2001.
[5] I. Ortlepp u.a.: „Ultrathin transparent photodetector for use in Standing-Wave-Interferometer" in XXI IMEKO World Congress "Measurement in Research and Industry" 30. August - 4 September, Prag, S. 1519-1524, 2015.

[6] O. Wiener: „Stehende Lichtwellen und die Schwingungsrichtung polarisirten Lichtes" in Ann. Phys., 276: S.203-243, 1890. doi: 10.1002/andp.18902760603

[7] H. Büchner: „Stehende-Wellen-Interferometer zur Messung von optischen Gangunterschieden." Deutsches Patent DE 3300369, 1983.

[8] E. W. Silvertooth und S. F. Jacobs: „Standing wave sensor" in Appl. Opt. 22, S. 1274-1275, 1983. doi: 10.1364/AO.22.001274

[9] H. Kung, S. Bhalotra, J. Mansell, und D. Miller: "Compact transform spectrometer based on sampling a standing wave." in Red, 100.150, S. 200, 2000.

[10] M. Sasaki, X. Mi, und K. Hane: „Standing wave detection and interferometer application using a photodiode thinner than optical wavelength." in Applied Physics Letters, 75.14, S. 2008-2010, 1999. doi: 10.1063/1.124898

[11] Y. Li, X. Mi, M. Sasaki und K. Hane: „Precision optical displacement sensor based on ultra-thin film photodiode type optical interferometers." in Measurement Science and Technology, 14(4), S. 479, 2003.

[12] H. J. Büchner, H. Stiebig, V. Mandryka, E. Bunte und G. Jäger: „An optical standing-wave interferometer for displacement measurements" in Measurement science and technology, 14(3), S. 311, 2003.

[13] E. Bunte, K. Jun, und H. Stiebig: „Micro interferometer based on amorphous silicon nanosensor." in Journal of Optoelectronics and Advanced Materials, 7.4, S. 1899-1907, 2005.

[14] V. Mandryka, H. Büchner und G. Jäger: „Design aspects in the development of a standing wave interferometer" in Proc. SPIE 5457, Optical Metrology in Production Engineering, S. 175, 2004. doi:10.1117/12.546708

[15] H. Büchner, E. Bunte, V. Mandryka, H. Stiebig und G. Jäger: „Standing-wave interferometer based on partially transparent photodiodes" in Proc. SPIE 5144, Optical Measurement Systems for Industrial Inspection III, S.218, 2003. doi:10.1117/12.499902

[16] M. HOLÁ u. a.: „Nanopositioning with detection of a standing wave." in NANOCON 2013. International Conference /5., 2013.

[17] J. Lazar u.a.: „Interferometry with Stabilization of Atmospheric Wavelength." in X IMEKO TC14 Symposium on Laser Metrology for Precision Measurement and Inspection in Industry, Braunschweig, Germany, Sept. 2011. 